\title{
La huella del neumático como soporte publicitario. El caso de Michelin y las batallas comerciales entre las compañías pioneras del sector (1904-1916)
}

\author{
Pau MEDRANO BIGAS ${ }^{1}$ \\ Universidad de Barcelona
}

\begin{abstract}
RESUMEN:
La encarnizada lucha tecnológica y comercial entre las compañías pioneras del sector del neumático, estrechamente ligada a la librada entre los constructores de automóviles, fue especialmente intensa en las dos primeras décadas del siglo XX y tuvo su reflejo en las distintas estrategias publicitarias adoptadas. El cambio de siglo dejó atrás la era de la bicicleta para desplazar el foco a la emergente industria del motor. Compañías como las francesas Michelin y Bergougnan, la alemana Continental, la italiana Pirelli, las británicas Dunlop y North British o las americanas US Rubber, Goodyear, Goodrich y Firestone, iniciaron politicas de expansión internacional al mismo tiempo que -ante la invasión de firmas rivales foráneas- reforzaban su presencia local. La aparición en 1904 de las novedosas cubiertas neumáticas enteramente de goma y con dibujo antideslizante en su suela -lisa hasta el momento- desató una auténtica batalla comercial entre sus valedores y sus detractores. La beligerante política de comunicación de la firma Michelin fue especialmente significativa, y la utilización intensiva de la publicidad comparativa como recurso desató la airada respuesta de sus competidores.
\end{abstract}

PALABRAS CLAVE: Publicidad comparativa; Industria del neumático; Michelin, Dunlop; Bibendum.

TITLE: The tire track as an advertising medium. The case of Michelin and the trade wars between the pioneers in the sector (1904-1916)

\begin{abstract}
:
The fierce technological and commercial competition between the pioneers in tire production, closely linked to the rivalry between automobile producers, was especially intense in the first two decades of the twentieth century and is reflected in the different advertising strategies that the various firms adopted. The new century marked the end of the era of the bicycle and the emergence of the motorcar industry. Companies like Michelin and Bergougnan in France, Continental in Germany, Pirelli in Italy, Dunlop and North British in the UK, and US Rubber, Goodyear, Goodrich and Firestone in the US, all moved into the international market and also fought to strengthen their local presence in the face of competition from their foreign rivals. In 1904 the appearance of the new coverings made entirely of rubber and bearing non-skid tread (tires until that time had been plain) sparked off a full-scale trade war between the new tires' supporters and
\end{abstract}

1 Profesor asociado del Departamento de Diseño e Imagen de la Facultad de Bellas Artes de la Universidad de Barcelona. Licenciado en Bellas Artes, especialidad de Diseño. Ejerciendo profesionalmente en el sector editorial como diseñador gráfico y director de arte durante los últimos dieciséis años, y en la actualidad compaginando la actividad docente con la de investigador en la Tesis Doctoral sobre diseño, ilustración y publicidad en las compañías pioneras del neumático (1900-1930), bajo la dirección de la Doctora Anna Calvera Sagué. Email: pau.medrano.bigas@ub.edu 
detractors. The aggressive publicity campaign launched by Michelin was particularly significant, and the intensive use of comparative advertising met with an angry response from its competitors.

KEY WORDS: Comparative advertising; Tire industry; Michelin; Dunlop; Bibendum.

Desde su iniciación en el negocio del neumático en 1896 hasta la primera década de 1900, las ambiciones de la compañía Michelin et Cie. y el contexto del mercado habían cambiado sustancialmente. La empresa no solo luchaba por el negocio continental desde su baluarte francés de Clermont-Ferrand y sus centros estratégicos en Reino Unido -con agencia en Londres desde 1905-, Italia -la fábrica de Turín, puesta en marcha en 1907- y los Estados Unidos -con fábrica en Milltown, New Jersey, operativa desde 1908- y sus distintas delegaciones en otros países europeos ${ }^{1}$; también debía coordinar sus estrategias en un mercado global que incluía territorios como el del continente americano o Australia y Nueva Zelanda.

Esta voluntad de expansión internacional, así como las fricciones producidas en el encaje y adecuación de su política comunicacional a la particularidad de los mercados locales, se puede apreciar en el extenso legado patrimonial de anuncios y elementos publicitarios generados en esa época.

La publicidad comparativa fue una de las estrategias más significativas utilizadas en la política comunicacional de Michelin para competir en los distintos escenarios. La singularidad, virulencia y notoriedad de las campañas para posicionarse con ventaja frente a sus rivales es el objeto de estudio de este trabajo.

La Michelin et Cie. estaba, de facto, bajo una dirección bicéfala. Por una parte el hermano pequeño, Edouard, estaba a cargo de la gestión de los procesos de fabricación en la factoría de Clermont-Ferrand; por la otra el hermano mayor, André, comandaba el aspecto comercial -que incluía el servicio de comunicación y publicidad- instalado desde el año 1900 en la sede del número 105 del boulevard Pereire, en la capital parisina. Desde el «Bureau de Paris» se encargaban las ilustraciones a distintos artistas free lance, que eran más tarde complementadas con los mensajes tipográficos para, finalmente, ser remitidas a las distintas publicaciones en las que se había contratado la correspondiente inserción publicitaria. Los departamentos homólogos de las filiales en el extranjero estuvieron bajo el control de la casa madre, recibiendo directrices y material gráfico para la elaboración de sus campañas, siempre supervisadas.

Los mercados menores europeos, próximos y dimensionados -como Alemania, Bélgica, España u Holanda- utilizaron una publicidad basada en la adaptación de

1 Además de la fuerte presencia en Gran Bretaña, Michelin controlaba agencias propias en Bélgica, Holanda y Austria desde 1900, y en Suiza, Italia, Alemania y España desde 1901. La delegación italiana contaba en 1913 en su fábrica con más de 2.000 empleados - una cifra similar a la de la plantilla de la fábrica de la filial americana-, respondiendo a la evolución de la Fiat y dedicada también a la exportación a Gran Bretaña, Alemania y Austria. 
clichés elaborados originalmente para el mercado francés por ilustradores locales como O'Galop, Chenet o Cousyn. En cambio, las tres filiales estratégicas implantadas en Reino Unido, Italia y los Estados Unidos tuvieron un cierto margen de maniobra durante estos primeros años.

Se dispone de muy poca información sobre las agencias, escritores, directores de arte, ilustradores y diseñadores que participaron en cada caso y, especialmente, existe un gran vacío en cuanto al modo de operar de la Michelin Tyre Company de Londres, aunque es evidente que la homogeneidad en su política comunicativa precisó del control de una experimentada agencia local. En este caso concreto, a pesar de la intensa producción publicitaria -especialmente en prensa- ninguno de los anuncios, ni de las numerosas ilustraciones producidas exprofeso desde y para el mercado inglés, aparece firmado por sus autores o por una agencia.

\section{De lo liso y lo rugoso}

Como sucede en otros campos de la industria sometidos a una feroz competencia, los logros tecnológicos de probada solvencia obtenidos por una determinada compañía de neumáticos eran rápidamente mimetizados por el resto. La superfície de la banda de rodadura de las ruedas macizas y neumáticas de los diferentes fabricantes en los primeros años era lisa, a lo sumo algunos modelos incorporaban tímidas estías; su sección transversal presentaba, además, un perfil redondeado [round tread], por lo que sólo una parte de la suela tenía un contacto uniforme con el suelo. Todo ello reportaba menos rozamiento $\mathrm{y}$, por tanto, mayor velocidad... pero también menor agarre.

En el año 1904 la compañía alemana Continental, seguida de cerca por Michelin, apostó por la banda de rodadura plana [flat tread] en las ruedas lisas, una tecnología que permitía una zona de contacto más amplia con la superficie de la carretera, una mejor tracción y un desgaste más homogéneo. Apenas dos años más tarde la mayor parte de los fabricantes -incluidos los americanos G \& J y Diamond- las habían asimilado y estandarizaron su uso.

La superfície lisa seguía dificultando la conducción provocando derrapajes y deslizamientos, lo que demandaba en ocasiones el uso de cadenas. Por ese motivo se buscó una solución para dotar a los neumáticos de una suela antideslizante -en América conocida como non-skid tread-, integrando las ventajas de las cadenas metálicas en la propia cubierta de la rueda.

La respuesta a estos inconvenientes fue el desarrollo en 1905-1906 de tiras de cuero integrando rivetes de metal -steel studded treads- que se encolaban a las bandas de rodadura de las cubiertas lisas, apareciendo en el mercado los neumáticos denominados armored tires, como la «Semelle» de Michelin, la «Rouge Ferré» de Continental o el «Neroferrato» de Pirelli (1907). Esta propuesta fue rápidamente adoptada por la mayor parte de firmas. 


\section{Antideslizantes con acento inglés}

A pesar de su aceptación, este tipo de suelas presentaba ciertos inconvenientes como demuestran las medidas promulgadas en el Reino Unido para limitar su uso o gravarlo con impuestos especiales. Con la mejora de las carreteras y la pavimentación de los trazados urbanos, el agarre y tracción necesarios que aportaban las steel studded treads en carreteras de tierra se transformó en erosión y desgaste de las nuevas superficies, deterioradas por la acción del metal sobre el firme. El debate que se generó al respecto ocupó una serie de noticias aparecidas a lo largo de 1907 y 1908 en el periódico The Times ${ }^{2}$.

Precisamente en ese país fue donde se plantearon algunas de las alternativas tecnológicas a este tipo de neumáticos. La compañía Dunlop presentó en la primera edición del Stanley Automobile Show, celebrado en enero de 1903 en el Earl's Court de Londres, su nuevo modelo de neumáticos antideslizantes enteramente de goma «Grooved Tread»-comercializados en Francia como «Le Cannelé»-, que tenían una serie de surcos o muescas acanaladas transversales y paralelas repetidas a lo largo de la banda de rodadura. Este modelo fue ganando terreno hasta convertirse en el más característico de la firma, y fue comercializado también en los mercados internacionales hasta bien entrados los años veinte.

Pero una tecnología distinta de suela antideslizante creada al otro lado del Atlántico acabó imponiéndose. En Estados Unidos Charles J. Bailey, con su cubierta «Bailey Won’t Slip» de 1904, apostó también por eliminar el metal y substituirlo por goma. A diferencia de las hendiduras propias de las cubiertas acanaladas, las suelas Bailey estaban formadas por hileras de ribetes de goma proyectados hacia el exterior, diseñados para crear una superficie rugosa. Esas protuberancias o tacos antideslizantes cilíndricos estaban generados en el mismo molde de cocción del neumático, lo que singularizaba a esta suela antideslizante enteramente de goma, denominada genericamente rubber non-slip tread o rubber non-skid tread ${ }^{3}$.

Hacia 1910 la mayor parte de los fabricantes americanos se habían decantado por las suelas tipo Bailey y la tendencia se extendió a Europa.

En 1912 se estimaba que un 40\% de las cubiertas neumáticas del mercado americano eran modelos con suelas antideslizantes -sumando los dos tipos básicos steel studded y rubber non-skid-, que costaban entre un 18-20\% más que los neu-

2 Según las noticias publicadas en The Times: «Automobile notes», 29 de enero de 1907; «Motor-car traffic and road maintenance», 22 de agosto de 1907; «Motor engineering», 4 de diciembre de 1907; «Side slip», 23 de junio de 1908; «The upkeep of roads», 13 de agosto de 1908; «Road upkeep and taxation», 10 de noviembre de 1908.

3 En los artículos de las revistas del motor estadounidenses de la época se solía dividir en dos tipologías a las cubiertas neumáticas, según la forma de la suela: las depressed tread, es decir, las que presentaban canales o hendiduras por debajo del nivel base de la banda de rodadura, y las raised tread, aquellas que proyectaban tacos de goma. 
máticos lisos, en parte por el aporte extra de caucho y material ${ }^{4}$. A las puertas de la Primera Guerra Mundial la tecnología de suelas antideslizantes de goma había substituido a los neumáticos lisos y a los que utilizaban refuerzos de metal, convirtiéndose en el estándar internacional de prácticamente todos los fabricantes de neumáticos.

\section{Publicidad en-cubierta}

En las cubiertas antideslizantes se aprovechó la extrusión de caucho extra para componer distintos dibujos, que a menudo reproducían los logotipos e iniciales de cada compañía. La construcción geométrica y disposición de los tacos -a menudo caprichosa- iba encaminada en la mayoría de los casos a conseguir un dibujo diferencial propio, un diseño único y patentable, más que a resolver científicamente los problemas de deslizamiento y agarre del neumático.

La huella dejada por las ruedas sobre el suelo mojado o enfangado constituía, además, un reclamo publicitario de la marca. Eslóganes, nombres comerciales, logotipos y símbolos no solo competían en los anuncios de prensa o en las vallas junto a la carretera, también se estampaban en las calles y caminos en una continua lucha publicitaria por el centímetro cuadrado de calzada. Uno de los diseños más notorios fue el de los neumáticos «NON SKID» [no derrapan, no se deslizan] de Firestone creados en 1908, que mostraban estas dos palabras - escritas en gruesas letras capitales- como motivo repetido en franjas diagonales paralelas y volumétricas a lo largo de la banda de rodadura (fig. 5).

En realidad estas suelas eran la adaptación de un curioso invento de principios de siglo, utilizado también en Estados Unidos en distintas acciones publicitarias y específicamente en las elecciones presidenciales de 1904 entre los candidatos Parker y Roosevelt. En Francia era conocido como la bottine imprimeuse, y consistía en una serie de tacos de goma individuales, cada uno de los cuales correspondía a las distintas letras del alfabeto. Estas piezas se engarzaban en unas guías o cables que iban sujetos a la banda de rodadura de la rueda; combinando las diferentes matrices -al estilo de los tipos de fundición-, se componían palabras y frases. Estas ruedas se montaban en vehículos como las ligeras voiturettes, actuando como un enorme sello de caucho circular: primero se impregnaba su suela de tinta para que, al girar y entrar en contacto con el pavimento, estampara el mensaje tipográfico, repitiéndose continuamente el proceso mientras durara la marcha ${ }^{5}$.

4 «Non-Skid treads feature of 1912 tires», Motor Age, 25 de enero de 1912, 43; «Tires, proper use and care needed», The Automobile, 11 de enero de 1912, 179.

5 «La bottine imprimeuse», noticia publicada el 6 de octubre de 1904 en el periódico La Liberté de Friburgo, Suiza. También se comenta el artefacto en un capítulo de la obra reseñada: BAUDIN, H. (1905). L'enseigne et l'affiche, Ginebra, Suiza, Impr. Atar. 


\section{A contracorriente}

Michelin desestimó sumarse a la revolución que se estaba gestando en tierras americanas e inglesas, defendiendo con empeño en todos los mercados internacionales y en el propio francés la tecnología de sus ruedas antideslizantes «Semelle» con rivetes de metal, y las «Compressed Tread»-denominadas «Carpe» en Francia-, con unos nervios y surcos continuos longitudinales en la banda de rodadura a modo de acordeón, que, al inflarse el neumático, en vez de expandirse se contraían formando una superficie más dura y resistente que la propia de las cubiertas lisas. La apuesta por mantener invariable su catálogo de neumáticos entre 1908 y 1915 fue decidida... y seguramente temeraria.

Pronto la Michelin et Cie. se sintió asediada por las emergentes suelas de caucho antideslizantes con dibujo, las grooved treads inglesas y las rubber non-skid treads americanas que iban ganando adeptos a un ritmo exponencial. La respuesta de la compañía fue sorprendente. Confiados en el resultado de las anteriores batallas libradas en Francia contra Dunlop y Continental, saldadas a menudo con éxito, decidieron mantenerse firmes en su posición e iniciar una agresiva campaña contra los nuevos neumáticos. Pero en un mercado globalizado su planteamiento debía atajar varios frentes: no se trataba de una mera refriega contra un único competidor. Oponerse a la nueva tecnología significaba enfrentarse en el mercado francés, británico, europeo y estadounidense -y en otros como el canadiense, el sudamericano, el australiano...- a la tendencia adoptada por la mayoría de las compañías.

En un error de cálculo, quizás subestimando el alcance real del cambio tecnológico y la dimensión de sus rivales, se iniciaron entre 1911 y 1913 punzantes campañas en Francia (fig. 4), Gran Bretaña y en los Estados Unidos para desacreditar los nuevos neumáticos antideslizantes. Michelin partía con ventaja en Francia, su mercado natural; en Gran Bretaña, por contra, debía enfrentarse en territorio de Dunlop. Por último, no podía alzar su voz con autoridad en los Estados Unidos, pues allí sólo era una firma más en la larga lista de compañías medianas del sector, a gran distancia del ranking encabezado por las poderosas Big Four: US Rubber, Goodyear, Goodrich (que absorbió a Diamond en 1912) y Firestone, y otras destacadas como Fisk, Kelly o Miller. A principios de los años veinte se contabilizaban más de 270 industrias estadounidenses dedicadas a la fabricación de ruedas macizas y neumáticos de goma ${ }^{6}$.

6 BUENSTORF, G. Y KLEPPER, S. (2005): «The origin and location of entrants in the evolution of U. S. tire industry», The Papers on Economics and Evolution, Jena, Alemania, Evolutionary Economics GroupMax Planck Institute, 3. 


\section{Michelin vs Dunlop}

La rivalidad entre la francesa Michelin y la británica Dunlop se remonta a la última década del siglo XIX, en la que ambas pugnaban en sus respectivos países y en gran parte de Europa por el revolucionado mercado de la bicicleta. La Dunlop Tyre Company Limited se formó en 1889 por iniciativa del empresario y capitalista Harvey du Cros para explotar las patentes de John Boyd Dunlop sobre la tecnología del neumático. Dunlop, también socio del negocio en un principio, vendió sus participaciones a du Cros en 1894, que se hizo con el control total de la empresa.

En 1893 se creó la Compagnie Française des Pneumatiques Dunlop y en octubre de 1896 du Cros dio un paso más cuando, a través de una sociedad inversora en la que participaba, adquirió los dos mayores fabricantes de bicicletas de Francia, las firmas Clément y Gladiator, y la subsidiaria francesa de las bicicletas inglesas Humber, para calzar toda la producción con sus ruedas?

La aparición de los neumáticos desmontables para bicicletas de Michelin en 1891, un hito tecnológico y un éxito comercial, constituyó una importante cuña en el monopolio ejercido hasta entonces por Dunlop, y fue en 1896 cuando Michelin tomó definitivamente la iniciativa, con la comercialización del primer neumático para automóviles ${ }^{8}$. El desarrollo del vehículo a motor provocó que Gran Bretaña con una industria automovilística prácticamente inexistente- se viera invadida antes del cambio de siglo por coches básicamente franceses y alemanes equipados en origen con componentes como los neumáticos de Michelin. Dunlop, en cambio, no se decantó por el mundo del motor -su primer neumático para automóviles se introdujo en julio de 1901-, pero apostó por proteger estratégicamente su mercado adquiriendo los derechos de una serie de patentes estratégicas.

En abril de 1904, Michelin estableció una delegación en Londres, anticipándose a la cercana expiración de las patentes. El 7 de junio de 1905 se constituyó legalmente la Michelin Tyre Company Limited y estuvo presente -por primera vez con stand propio- en el prestigioso Olympia Motor Show, celebrado en el Agricultural Hall de la misma ciudad entre el 17 y el 25 de noviembre de 1905.

En 1907, el mercado británico se había convertido en el de mayor crecimiento en Europa debido a las importaciones de vehículos del continente y americanos, sumado al despertar de su propia industria: cerca de 53.000 vehículos motorizados registrados en Gran Bretaña, frente a los 31.000 en Francia o los 16.000 en Alemania ${ }^{9}$.

En febrero de 1908 la Michelin Tyre Company Ltd. obtuvo un reconocimiento de gran importancia -aprovechado promocionalmente, por supuesto-, la garantía

7 DumOND, L. (1996): L'industrie française du caoutchouc, 1828-1938. Analyse d'un secteur de production. Tesis doctoral, Universite Paris 7 Denis-Diderot, 579-580.

8 En Francia, la fecha de inicio de fabricación de neumáticos Dunlop para automóviles es 1902, a través de la Dunlop Pneumatic Tyre Compagnie (France) con fábrica en Argenteuil.

9 NibBlett, P. y ReynoldS, J. (2005): Michelin Centenary, 1905-2005. A celebration of Michelin's first hundred years in the british isles, Watford, Herfordshire, Michelin Travel Publications, 21-22. 
de proveedor de la Casa Real inglesa otorgada por el monarca Edward VII. Un año más tarde, el 20 de enero de 1911, inauguró un singular edificio conocido como la Michelin House, de estilo Art Noveau y construido expresamente para albergar su nuevo cuartel general en Londres. La arquitectura y los elementos decorativos de la fachada, con paneles cerámicos policromados conmemorando los hitos de la historia corporativa en las competiciones automovilísticas, así como las impactantes vidrieras reproduciendo a la mascota corporativa -Bibendum, el hombre neumático- según los carteles originales de su creador el ilustrador francés Marius Rossillon «O'Galop», convertían a la edificación en un imponente y permanente reclamo publicitario ${ }^{10}$. Michelin se encontraba en una posición inmejorable para plantar cara a su principal competidor en su propio terreno, y esto se tradujo en la intensificación de las campañas publicitarias y en una acrecentada rivalidad.

\section{Lecciones de patriotismo}

Una de las primeras escaramuzas entre Michelin y Dunlop la acogió en 1900 la prensa francesa, en una serie de anuncios escritos en tono patriótico y firmados por Michelin en los que se argumentaba que, a pesar de la presencia de la Compagnie Française des Pneumatiques Dunlop, los productos Dunlop estaban en realidad fabricados en Gran Bretaña. La misma estrategia fue aplicada a su otro máximo rival europeo, la Continental alemana, que estaba bien implantada en Francia ${ }^{11}$.

La respuesta de Dunlop se reflejó en un anuncio en la prensa británica, aprovechando la celebración del prestigioso Olympia Motor Show de 1910 (fig. 1). En la escena, el personaje de representación popular patriótica británica John Bull sentado en una pila de neumáticos acanalados Dunlop- observa con una lupa a un diminuto Bibendum, en referencia al percentualmente escaso protagonismo de Michelin en la feria, en desigual competencia con la omnipresente Dunlop ${ }^{12}$.

En el primer trimestre de 1911, ya desde las dependencias de la Michelin House, el departamento de publicidad inició una provocadora campaña en defensa de sus modelos «Square Tread»y «Semelle»y dirigida contra las cubiertas antideslizantes enteramente de goma, especialmente las ruedas acanaladas de Dunlop. El texto de un anuncio publicado en el mes de marzo en el periódico The Times afirmaba:

10 Hitchmough, W. (1995): The Michelin building: a celebration of the reopen, Londres, Octopus Publishing/Reed Consumer Books.

11 DARmon, O. (1997): Le gran siècle de Bibendum, París, Editions Hoëbeke, 36. Por su parte, Harp recoge en su interesante libro varias disputas legales y publicitarias entre Michelin y Continental en el mercado francés; HARP. S. (2001). Marketing Michelin. Advertising and cultural identity in twentiethcentury France. Baltimore, The Johns Hopkins University Press, 70-71.

12 «When the truth is told», anuncio en The Autocar, 26 de noviembre de 1910. 
[...] el modelo «Square Tread» de Michelin no es un neumático de suela decorativa; no tiene un diseño escultural bonito en la suela; no tiene protuberancias o profundos canales que mermen el grosor de la goma y la deformen. Por tanto, presenta una superficie uniforme con la carretera, y el desgaste se distribuye homogéneamente. Además -y esto es lo más importante- usted no está pagando por el caucho que ha sido extraído y eliminado. Elaborar diseños y decoraciones en el caucho cuesta dinero, y los beneficios reales obtenidos son...? ${ }^{13}$

En septiembre de ese mismo año se volvió a incidir sobre el tema en un controvertido anuncio a toda página en la revista The Illustrated London News. Mostrando una pila de varios neumáticos con diferentes diseños de suela, se concluía:

Parece que [el resto de los fabricantes] están intentando complacer a los clientes del mismo modo en que lo haría un marchante de arte que, al mostrar una pintura con un marco vistoso, distrajera la atención sobre el valor real de la pintura a base de loar los méritos del marco. ¿Pero dónde reside el valor real?, ¿en la misma pintura o en el marco? El hecho de que exista una gran variedad de ruedas con suelas caprichosas prueba concluyentemente que ninguna de ellas es válida. Si una u otra fuera la correcta, debería ser adoptada universalmente por todos los fabricantes de cualquier parte del mundo, o todas son realmente innecesarias. [...] ¿No estoy en lo cierto cuando digo que en realidad son un gran bluf publicitario ${ }^{14}$

Y en una virulenta acometida, publicada en el número del mes de octubre de la revista del mundo del motor The Autocar:

«Ellos [refiriéndose a Dunlop] han descubierto que a base de gritar no han sacado ningún provecho $\mathrm{y}$, para intentar mantener una parte de los beneficios que temen perder, han adoptado una nueva táctica, que puede ser llamada como el proceso de vender ruedas entre un 12 y un 15 por ciento por encima del precio razonable. Asuma por un momento que usted fuera un fabricante que pretendiera hacer eso. Por supuesto, debería adornar el interior de los moldes con un diseño en relieve que le ahorrara goma [...]. Y bautizaría su nuevo producto con nombres decorativos: cubiertas de carreras extra grabadas a mano y cubiertas de turismo de trazado triple, o algo similar. Usted inauguraría una nueva columna en su listado de precios y los subiría un 12 , un 15 o un 20 por ciento ${ }^{15}$.

Pero fue iniciada la Primera Guerra Mundial cuando las posiciones se radicalizaron. Las compañías británicas Dunlop, Avon, Moseley, Wood-Milne, Henley, Beldam, Palmer o North British, insertaban anuncios patrióticos en la prensa abo-

\footnotetext{
13 «Bibendum Square Tread», anuncio en The Times, 10 de marzo de 1911.

14 «Bibendum on tyre fashion», anuncio publicado en The London Illustrated News, 9 de septiembre de 1911.

15 Anuncio en la revista británica The Autocar, octubre de 1911. El mismo texto, adaptado al mercado francés, se repite en «Peinture et sculpture pour pneumatiques», Automobile Club du Nord. Boletín oficial mensual de la Société Régionale d'Encouragement de l'Automobile-Club du Nord de la France, Roubaix, noviembre de 1911.
} 
gando por un consumo de los productos nacionales frente a la creciente importación de neumáticos franceses y americanos: los beneficios debían revertir en la propia industria para fortalecer financieramente a un país volcado en el esfuerzo bélico. Además, las compañías nacionales participaban suministrando ruedas al ejército británico y al de sus aliados, como el australiano.

La Michelin Tyre Company era considerada como una compañía extranjera -sus ruedas eran de importación, no tuvo fábrica inglesa hasta 1927- y consecuentemente no debía beneficiarse de la situación. La respuesta de Michelin frente a estas posiciones fue tajante al acusar de comportamiento antipatriótico y desleal a Dunlop, aunque sin nombrarla. En un anuncio publicado en septiembre de 1915 en The Times, un Bibendum sonriente encabezaba un largo texto, en el que denunciaba:

Un competidor, considerando que Michelin es una firma «extranjera», pues es francesa, ha proclamado que Michelin no ha contribuido en nada al esfuerzo bélico. Nos vemos forzados a responder que Michelin ha contribuido más al esfuerzo bélico que cualquier otro fabricante de neumáticos [...] Dejamos en todo caso que sea el público quien juzgue si este competidor, el cual antes de la guerra vendía en este país neumáticos «made in Germany» de una filial que al iniciarse la Guerra se declaró más alemana y patriótica que el más prominente de los fabricantes germanos, está autorizado para hablar de neumáticos «extranjeros» y para poner en duda el patriotismo de una firma francesa. ${ }^{16}$

Este torpedo dirigido a la línea de flotación de Dunlop hacía referencia a las actividades internacionales del fabricante británico iniciadas hacia 1889 con la finalidad de establecer agencias en América, Canadá, Bélgica, Francia... y Alemania. En este último país se creó en 1893 la sociedad limitada Deutsche Dunlop Gummi Compagnie, con fábrica en Hanau, al Este de Francfort, ciudad en la que se concentraba la industria germana de la bicicleta ${ }^{17}$. A partir de 1907 se constituyó en corporación, nutriendo al mercado británico con sus productos antes del estallido de la contienda.

\section{Tribunal de guerra}

El intercambio y la acumulación de reproches culminó finalmente en 1917 en un proceso legal, tras una demanda interpuesta por Dunlop. El denunciante reclamaba directamente daños y perjuicios a Michelin por los libelos en los anuncios insertados en los periódicos y revistas generalistas y en la prensa técnica del mundo del motor, en los

16 Anuncio en The Times, 3 de septiembre de 1915.

17 Erker, P. (2005): Vom nationalen zum globalen Wettbewerb. Die deutsche und die amerikanische Reifenindustrie im 19 und 20, Jahrhundert, Múnich, Schöningh, 150. 
que, según manifestaba, se atacaban su honestidad y buena fe. Michelin, en su defensa, alegaba que el comportamiento de su rival era similar al suyo ${ }^{18}$.

Especialmente hiriente fue el anuncio publicado en mayo de 1915 en periódicos y revistas inglesas y escocesas, en el que aparecía una ambulancia militar británica con una rueda pinchada (fig. 2). En medio de la batalla, un soldado se esforzaba en accionar el gato para cambiarla. A su lado, un complaciente Bibendum ofrecía como recambio uno de los anillos de su cuerpo al aliviado mecánico y al alborozado conductor. El eslogan bajo la ilustración era The Rib of Life, traducible literalmente por «la costilla de la vida»-Bibendum extraía el neumático de su caja torácica- aunque rib también refiere a piezas o nervios estructurales curvos en una construcción, así como a las líneas de trama paralelas en un estilo de costura. La rueda dañada retrataba claramente el reconocible modelo «Grooved Tread» de Dunlop, que en 1917 constituía el 50\% de los neumáticos fabricados por la compañía, y que era suministrado habitualmente a los vehículos militares y sanitarios del ejército británico.

Así que, en realidad, el mensaje transmitido era que los neumáticos Michelin «Compressed Tread» eran la salvación, frente a los neumáticos acanalados de Dunlop, que ponían en peligro la vida de los soldados. En este anuncio -según los demandantes- Michelin, con sus «ruedas de la vida», calificaba por comparación a los de Dunlop como «ruedas de la muerte».

Para el juez y el jurado el asunto no era si se trataba de una simple crítica o descalificación de los productos del rival en una reñida disputa comercial, sino si se habían producido imputaciones que atentaran contra la honestidad de cada compañía. El juez transmitió dos preguntas al jurado que podían esclarecer el dilema:

1. Pregunta: ¿Decían los acusados [Michelin] en sus anuncios más que lo razonablemente necesario para proteger sus intereses? Respuesta: Sí.

2. Pregunta: ¿Actuaron los acusados con malicia, es decir, por algún motivo indirecto distinto del de proteger sus intereses? Respuesta: $\mathrm{No}^{19}$.

El juicio se alargó durante siete días y finalmente se dictó sentencia el 21 de febrero de 1917. El veredicto del jurado estipulaba el pago de $£ 1.000$ de Michelin a Dunlop por daños y perjuicios... y también castigaba a Dunlop a pagar $£ 750$ a Michelin por el mismo motivo.

18 El desarrollo del proceso pudo seguirse en las interesantes y detalladas crónicas sobre el asunto, publicadas en el diario The Times los días 13, 14, 15, 20 y 21 de febrero de 1917. También otras publicaciones se hicieron eco del caso, como la británica The India Rubber Journal, con sendos artículos el 17 y el 24 de febrero o la americana The India Rubber World, en la noticia «Judicial decision» publicada el 1 de abril de 1917, 399.

19 «The motor tyres libel action: result», The Times, 21 de febrero de 1917. 


\section{Contra los neumáticos esculpidos americanos}

La implantación de Michelin en Estados Unidos, a través de la Michelin Tire Company con sede administrativa y fábrica en Milltown, New Jersey, era ya un hecho a principios de 1908. Los «Compressed Tread»-comercializados en Francia con el nombre de «Carpe» y en el mercado británico como «Square Tread»- fueron los primeros neumáticos producidos por Michelin en su factoría americana, que inauguró la producción hacia marzo de 1908. El gran despliegue publicitario anunciando el modelo «Semelle» en la prensa estadounidense de 1910-1912 da a entender que fue en esos años cuando la fábrica de Milltown estuvo finalmente capacitada para asumir la fabricación íntegra y masiva de ese modelo concreto.

La producción publicitaria de Michelin sufrió un bajón importante en 1913, y fue prácticamente inexistente en 1914 y 1915. Su ausencia fue especialmente acusada en los periódicos y en las revistas del sector del motor donde solía anunciarse habitualmente ${ }^{20}$. Aunque los Estados Unidos se mantuvo neutral en los primeros años de la Gran Guerra, es probable que la comunicación entre la delegación americana de Michelin y la casa madre francesa -así como con la Michelin Tyre de Gran Bretaña- quedara afectada; lo mismo debió ocurrir con el soporte financiero necesario para mantener las actividades promocionales.

En este contexto precario, Michelin trasladó al mercado estadounidense la campaña contra los neumáticos antideslizantes que había llevado a cabo en Europa. La estrategia -iniciada a finales de 1913 y desarrollada a lo largo de 1914- se basó especialmente en la distribución por correo de folletos promocionales, impresos a dos tintas, a doble cara y plegados generalmente en díptico o tríptico para adecuarse al alargado formato de sobre americano. Los textos de estos prospectos incidían en la descalificación de los neumáticos con cubiertas antideslizantes de goma que, según se explicaba, «incrementan sin motivo el precio en unos $\$ 5.00$ extra» y, por contra, «al utilizar los neumáticos «Plain Tread» de Michelin el ahorro es de un 17\%».

En estos panfletos se recreaba la situación de los usuarios de neumáticos engañados y exprimidos utilizando un curioso personaje (fig. 3). Se trataba de un orondo hombre de negocios de mirada inquisidora y rasgos duros, trajeado, con sombrero de copa y un cigarro en la boca, la encarnación estereotipada del empresario, del magnate fabricante de neumáticos que no dudaba en hacer su fortuna a costa de la buena fe de los clientes $^{21}$. En este caso el cilindro del sombrero de copa estaba formado por una pila de los más populares modelos de cubiertas del mercado norteamericano -el diseño es incon-

20 Medrano Bigas, P. (2005). «Los años olvidados. La etapa americana de Michelin en Milltown, 1907-1929. Nunc est bibendum !!.. Un mito gráfico desde 1898», libro-catálogo de la exposición itinerante del mismo nombre, Valladolid, Michelin España Portugal.

21 El sombrero refiere a una popular frase inglesa: be talking through your hat [estar hablando a través del sombrero], que se aplica a alguien cuando opina de un tema sin conocimiento de causa o cuando defiende que algo es cierto cuando en realidad no lo es. Se sugería, por tanto, que los argumentos en defensa de los neumáticos con dibujo antideslizante escondían realmente un engaño al consumidor. 
fundible, mostrando los modelos «Non Skid» de Firestone, «Nobby» $\mathrm{y}$ «Chain» de US Rubber, «Staggard» de Republic, «Zig-Zag» de Lee...

En ese individuo podrían haberse visto retratados alguno de los fundadores y directivos de las compañías americanas que apostaban - la gran mayoría- por los neumáticos antideslizantes con dibujo; podrían también haberse sentido insultados o tratados de forma despectiva... pero no hubo ninguna respuesta al respecto, en un acto de indiferencia que demostraba el poco calado y lo inadecuado de la política de Michelin ante la evidencia de las tendencias del mercado. Es más que probable que aquellos que podían haberse sentido aludidos consideraran una pérdida de tiempo y esfuerzo contestar a ese tipo de argumentos propagandísticos.

La publicidad comparativa constituyó un ariete básico en las estrategias de Michelin para preservar y conquistar mercados en territorios europeos. En los Estados Unidos, sin embargo -y a pesar de la feroz competencia desatada-, este tipo de publicidad no fue utilizado por ninguna compañía del sector. Las firmas del neumático norteamericanas centraron sus esfuerzos en afianzar su presencia, desarrollar innovaciones tecnológicas y extender su red comercial. Sus anuncios glosaban las virtudes de las propias compañías y sus productos, sin perder tiempo ni espacio - unos bienes valiosos- en enzarzarse en disputas públicas en las que, finalmente, se otorgaba al rival un protagonismo contraproducente.

\section{Una solución universal}

El 28 de septiembre de 1915, la United States Patent Office otorgó la patente número 47.892 de un nuevo diseño de cubierta para ruedas neumáticas, a nombre de Jules Hauvette Michelin, director de la Michelin Tire Company de Milltown y sobrino de Eduard y André Michelin, al frente de la casa madre francesa. Se trataba del denominado UT, «Universal Tread» [la suela universal], el primer neumático antideslizante Michelin enteramente de goma y con dibujo en la banda de rodadura, la apuesta para competir en el reñido mercado americano. Las otras compañías partían con cierta ventaja, bien posicionadas con sus modelos de antideslizantes presentes en el mercado entre tres y cuatro años antes de que Michelin decidiera, finalmente, apostar por los suyos propios ${ }^{22}$.

La cubierta «Universal Tread», según se explicaba en distintas notas de pren$\mathrm{sa}^{23}$, no se basaba en los antideslizantes con proyecciones de goma -como las sue-

22 El neumático «Universal Tread» fue también fabricado en 1917 en Francia y comercializado bajo el nombre de RU «Roulement Universal» y «Pneu Moderne», con un aspecto novedoso para los fieles consumidores de los productos Michelin con sus suelas esculpidas y un color oscuro característico -hasta el momento las cubiertas eran de un color gris claro-, fruto de aplicar por primera vez el negro de carbón como agente fortalecedor a la mezcla del caucho.

23 «Michelin brings out non-skid», The Automobile, 14 de octubre de 1915; «New Michelin tire produced», The Indianapolis Sunday Star, 17 de octubre de 1915; «Michelin develops new type of tire», La 
las Bayley- ni en los acanalados que utilizaban hendiduras, sino en una banda de rodadura plana formada por un nervio central como eje para la alineación vertical compacta de una serie repetitiva de gruesas letras capitales con la inicial «M» de Michelin.

No deja de sorprender que, después de volcarse en la descalificación del «decorativismo inútil» empleado por los rivales en el diseño de sus suelas neumáticas, el motivo gráfico escogido por Michelin resultara ser la inicial del nombre de la firma.

Con un cierto tono irónico la revista americana The India Rubber World, portavoz de la industria del sector del caucho, publicó a principios de 1916 una breve noticia sobre los nuevos neumáticos, zanjando definitivamente la polémica:

\begin{abstract}
Durante los pasados años las suelas antideslizantes [...] fueron productos aborrecidos por la importante compañía Michelin. Las llamaron suelas «esculpidas» y las satirizaron ingeniosamente en prosa y en verso. [...] Sin embargo, cada compañía de neumáticos ofrecía su propia suela antideslizante y a pesar de eso otras nuevas compañías añadieron las suyas, de manera que parecía que se habían agotado todos los posibles diseños. Entonces, y solo entonces, la compañía Michelin -quizás aceptando aquello que sabía desde hacía tiempo, que los usuarios de neumáticos reclamaban antideslizantes-, lanzó su propio modelo [...]. La compañía Michelin, de manera eficaz y elegante, se rindió a lo inevitable ${ }^{24}$.
\end{abstract}

\title{
10. Conclusión
}

Las estrategias de comunicación corporativa y publicitaria empleadas por las compañías de neumáticos de principios del siglo pasado son el reflejo de una época de virulenta competitividad. Las batallas tecnológicas y comerciales en los años previos a la Primera Guerra Mundial se reflejaron en una publicidad teñidas de tintes patrióticos y de descalificaciones, tanto entre las propias firmas adscritas al frente aliado -Michelin y Dunlop- como contra aquellas pertenecientes al bando contrario -como la alemana Continental-.

Gran parte de los reproches y acusaciones desembocaron en procesos judiciales en los que hubo vencedores y vencidos. La historia corporativa de cada compañía, escrita al dictado de los propios intereses, se ha encargado de corregir, resaltar o eliminar de la memoria colectiva aquellos pasajes comprometedores. La labor de investigación y rescate de estos sucesos y la experiencia que aportan nos permite reflexionar sobre la pertinencia y eficacia de una publicidad comparativa muy alejada de la que hoy en día conocemos, sometida a regulación y actuando con tiento dentro de los límites de lo «políticamente correcto».

Crosse Tribune, 27 de noviembre de 1915; «The Michelin tire», The Graham Guardian, 1 de junio de 1917; «The Michelin Tire Plant», The Rubber Age and Tire News, 25 de agosto de 1917, 447.

24 «The triumph of "sculptured tread"». The India Rubber World, 1 de febrero de 1916. 


\section{Referencias bibliográficas}

BAUDIn, H. (1905): L'enseigne et l'affiche, Ginebra, Suiza, Impr. Atar.

BUENSTORF, G. Y KLEPPER, S. (2005): «The origin and location of entrants in the evolution of U. S. tire industry», The Papers on Economics and Evolution, Jena, Alemania, Evolutionary Economics Group-Max Planck Institute.

DARMON, O. (1997) : Le gran siècle de Bibendum, París, Editions Hoëbeke.

DuMOND, L. (1996): L'industrie française du caoutchouc, 1828-1938. Analyse d'un secteur de production. Tesis doctoral, Universite Paris 7 Denis-Diderot.

ERKER, P. (2005): Vom nationalen zum globalen Wettbewerb. Die deutsche und die amerikanische Reifenindustrie im 19 und 20, Jahrhundert. Múnich, Schöningh.

HARP, S. (2001): Marketing Michelin. Advertising \& cultural identity in twentieth-century France, Baltimore, The Johns Hopkins University Press.

Hitchmough, W. (1995): The Michelin building: a celebration of the reopen, Londres, Octopus Publishing/Reed Consumer Books.

Medrano Bigas, P. (2005): «Los años olvidados. La etapa americana de Michelin en Milltown, 1907-1929. Nunc est bibendum !!.. Un mito gráfico desde 1898», libro-catálogo de la exposición itinerante del mismo nombre, Valladolid, Michelin España Portugal, 45-61.

NibBlett, P.; Reynolds, J. (2005): Michelin Centenary, 1905-2005. A celebration of Michelin's first hundred years in the british isles, Watford, Herfordshire, Michelin Travel Publications.

(1904): «La bottine imprimeuse», La Liberté, Friburgo, 6 de octubre de 1904.

(1907): «Automobile notes», The Times, Londres, 29 de enero de 1907.

(1907): «Motor-car traffic and road maintenance», The Times, Londres, 22 de agosto.

(1907): «Motor engineering», The Times, Londres, 4 de diciembre.

(1908): «Road upkeep and taxation», The Times, Londres, 10 de noviembre.

(1908): «Side slip», The Times, Londres, 23 de junio.

(1908): «The upkeep of roads», The Times, Londres, 13 de agosto.

(1910): «Bibendum Square Tread», The Times, Londres, 10 de marzo.

(1910): «When the truth is told», The Autocar, Londres, 26 de noviembre.

(1911): «Bibendum on tyre fashion», The London Illustrated News, Londres, 9 de septiembre.

(1911): «Peinture et sculpture pour pneumatiques», Automobile Club du Nord, Roubaix, noviembre. Boletín oficial mensual de la Société Régionale d'Encouragement de l'Automobile-Club du Nord de la France.

(1911): The Autocar, Londres, octubre.

(1912): «Non-Skid treads feature of 1912 tires», Motor Age, New York, 25 de enero, 43.

(1912): «Tires, proper use and care needed», The Automobile, New York, 11 de enero, 179.

(1915): The Times, Londres, 3 de septiembre.

(1915): «Michelin brings out non-skid», The Automobile, New York, 14 de octubre.

(1915): «Michelin develops new type of tire», La Crosse Tribune, La Crosse, Wisconsin, 27 de noviembre.

(1915): «New Michelin tire produced», The Indianapolis Sunday Star, Indianapolis, Indiana, 17 de octubre.

(1916): «The triumph of "sculptured' tread"», The India Rubber World, 1 de febrero. 
(1917): The India Rubber Journal, Londres, 17 y 24 de febrero.

(1917): The Times, Londres, 13, 14, 15, 20 y 21 de febrero.

(1917): «Judicial decision», The India Rubber World, New York, 1 de abril, 399.

(1917): «The Michelin tire», The Graham Guardian, Safford, Arizona, 1 de junio.

(1917): «The Michelin Tire Plant», The Rubber Age and Tire News, New York, 25 de agosto, 447.

(1917): «The motor tyres libel action: result», The Times, Londres, 21 de febrero.

Fig. 1. Anuncio de los neumáticos acanalados Dunlop publicado en la revista británica The Autocar, 26 de noviembre de 1910.

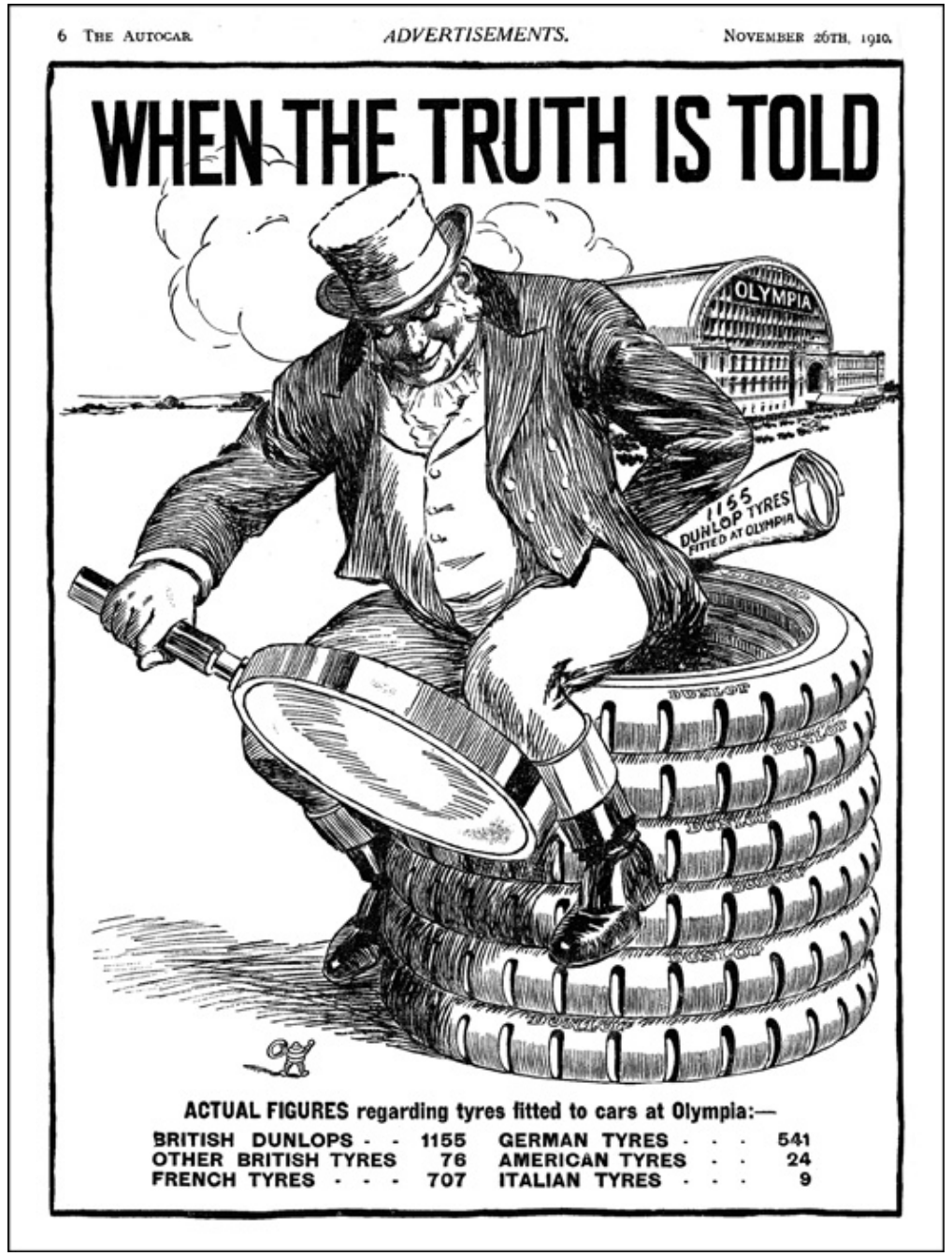


Fig. 2. Anuncio de los neumáticos Michelin

publicado en la revista británica The Illustrated London News, 8 de mayo de 1915.

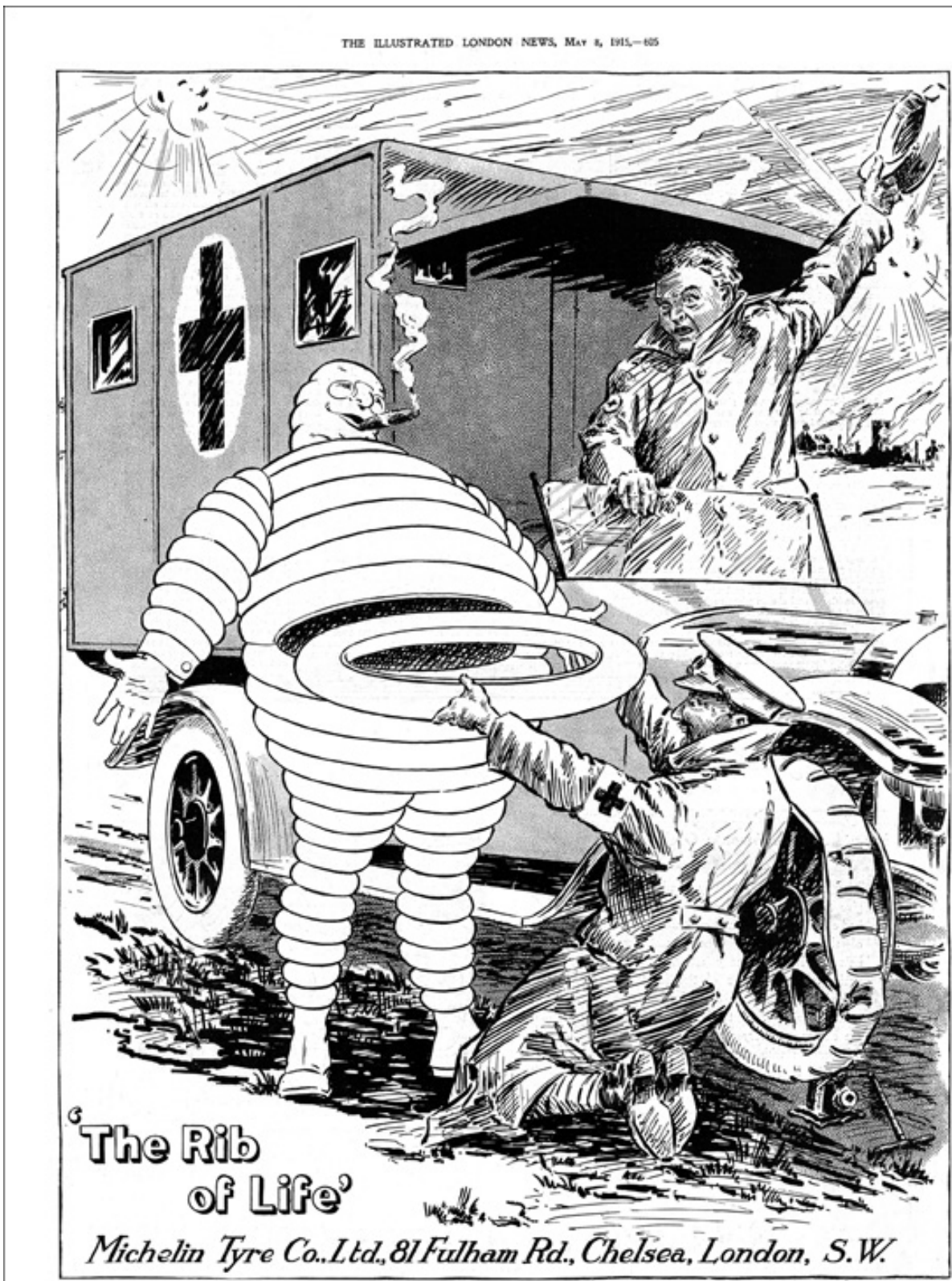


Fig. 3. Campaña americana de Michelin contra los neumáticos esculpidos.

Folleto publicitario y detalle de una de sus ilustraciones, 1913-1914.
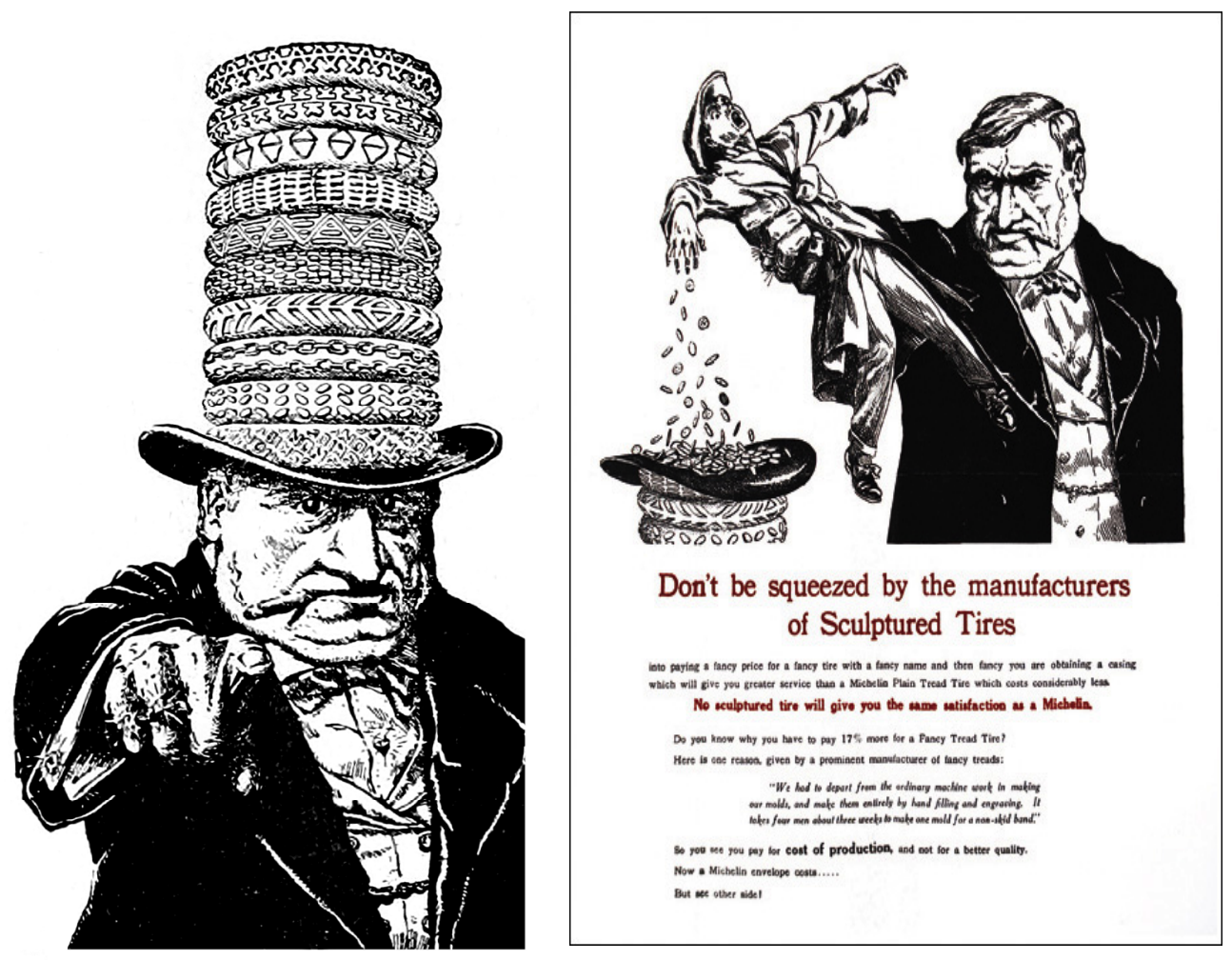
Fig. 4. Campaña francesa de Michelin contra los neumáticos esculpidos, mostrando a la mascota Bibendum a modo de Sansón derribando las columnas formadas por pilas de neumáticos de la competencia.

Anuncio publicado en la revista Femina en 1909. Ilustración obra de O'Galop.

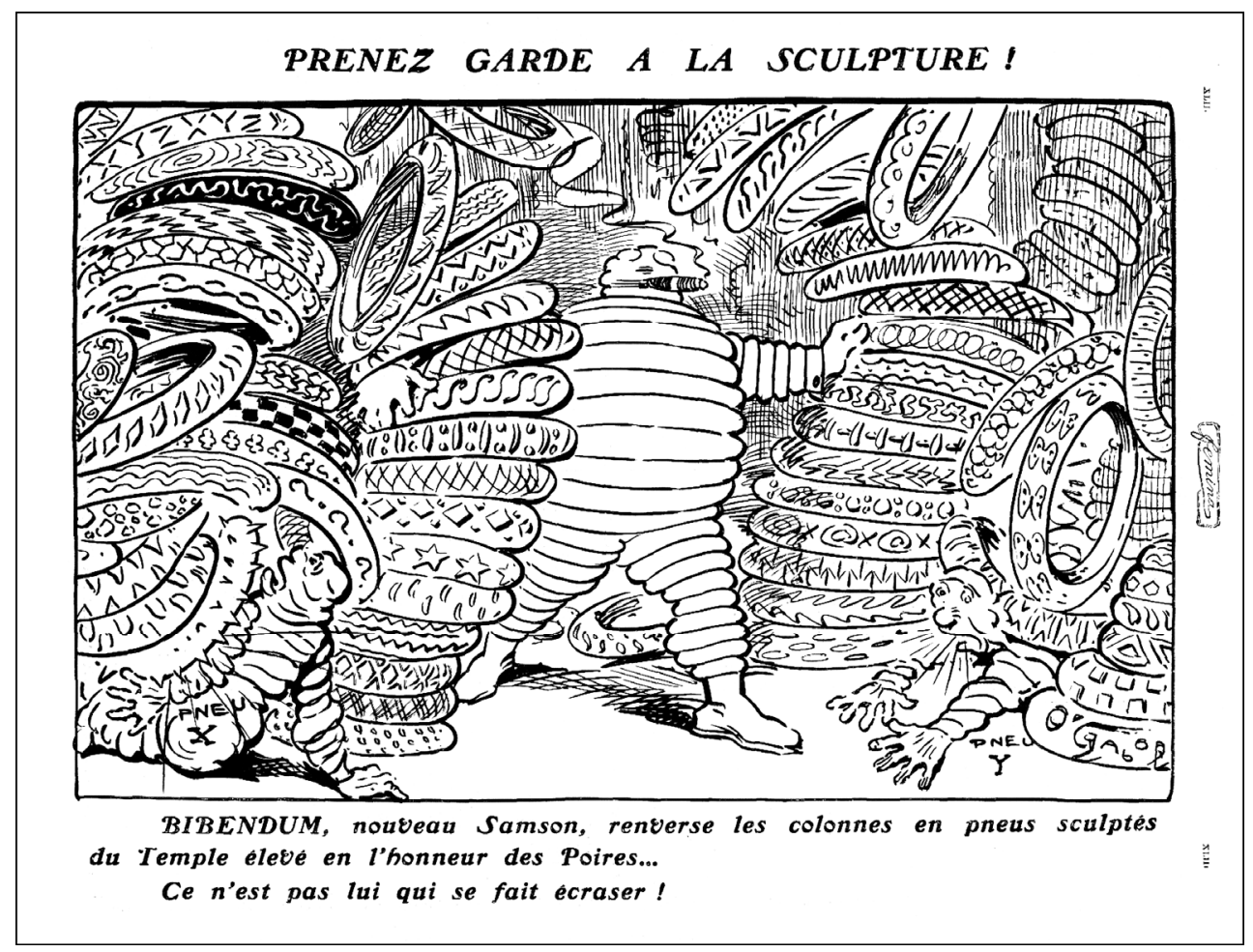


Fig. 5. Anuncio de los neumáticos antideslizantes de goma Firestone «Non-Skid», publicado en la revista americana The Literary Digest, 31 de marzo de 1917.

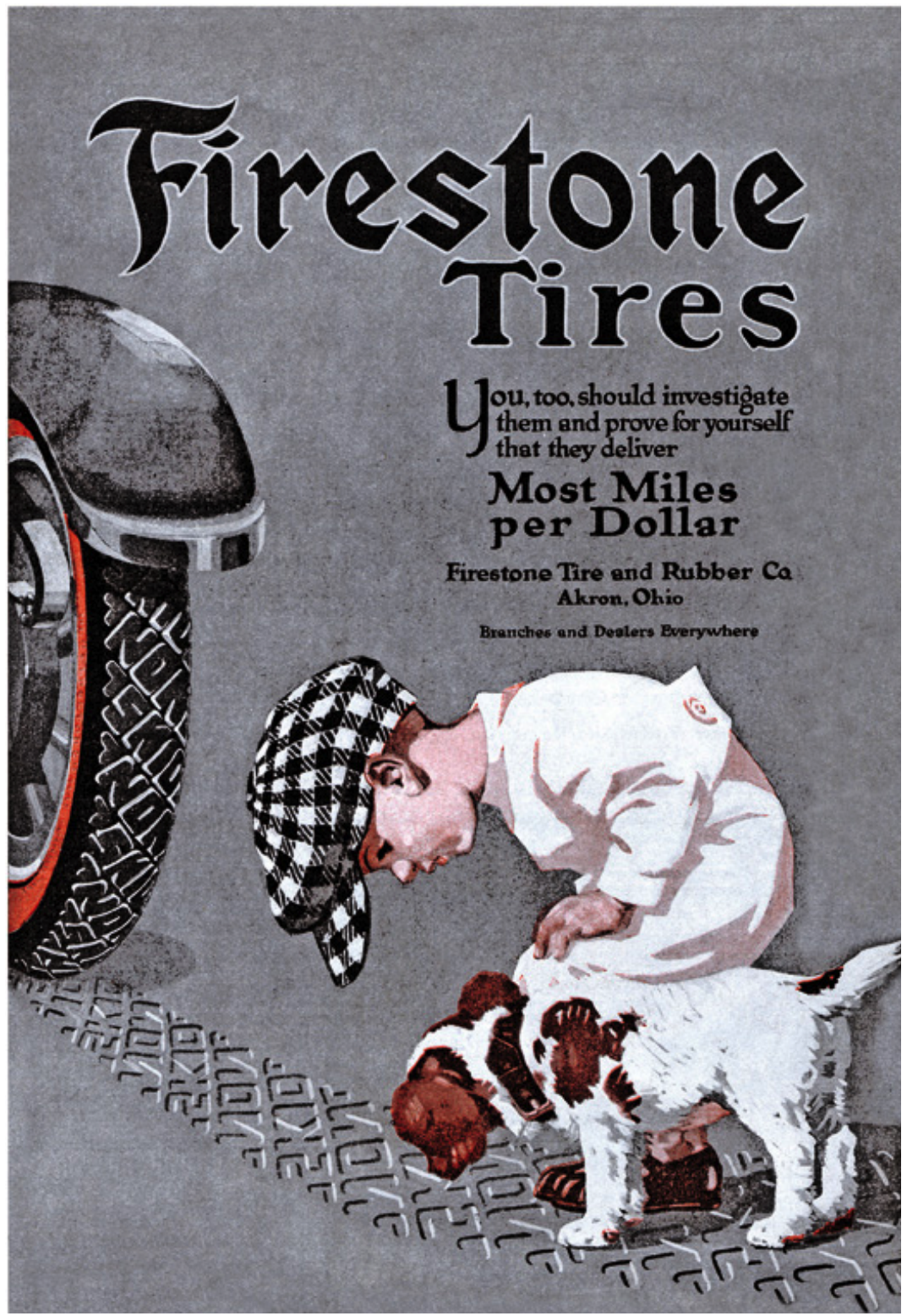

Recibido: 28 de febrero de 2011

Aceptado: 24 de septiembre de 2011 